\title{
Influence of playing style on the occurrence of missed shots in table tennis
}

\author{
Sho Tamaki', Kazuto Yoshida ${ }^{2,3}$ \\ ' Department of Sports and Health Science, Faculty of Human Health Sciences, Meio \\ University, Okinawa, Japan \\ ${ }^{2}$ College of Education, Academic Institute, Shizuoka University, Shizuoka, Japan \\ ${ }^{3}$ Graduate School of Health and Sports Science, Juntendo University, Chiba, Japan
}

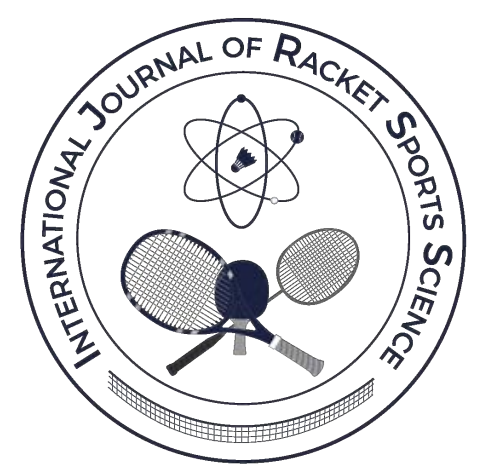

\begin{abstract}
Although the defensive style is competitive in modern table tennis, statistical analyses of it have been limited. The purpose of this study was to clarify the influence of playing style on the occurrence of missed shots, i.e. shots that resulted in a score for the opponent in table tennis. This study found that the defensive style decreases the incidence of missed shots, particularly after the fourth shot, although those of the second and the following shots also decrease. In matches played by a defensive player, missed shots were 6 to $8 \%$ less likely to occur than in matches between two offensive players. In addition, the defensive style reduces the likelihood of missed shots in a rally served by an offensive player, and the amount of reduction is about the same as in a rally served by a defensive player. By gender, male defensive players were less defensive than female. We also found it difficult to identify defensive players by the occurrence of missed shots because there might be players not categorised as having defensive style who were as defensive as defensive players.
\end{abstract}

Keywords: Table Tennis, Match Analysis, Performance Analysis, Defensive Player

Correspondence author: Sho Tamaki

E-mail: s.tamaki@meio-u.ac.jp

Cite this article as:

Tamaki, S., \& Yoshida, K. (2020). Influence of playing style on the occurrence of missed shots in table tennis.

International Journal of Racket Sports Science, 2(1), 32-41. 


\section{Introduction}

In table tennis, defensive players, also known as "choppers", are remarkably different from offensive players. Yuza et al. (1992) experimentally investigated the temporal, spatial, and physiological characteristics of playing styles by analysing six matches played by a defensive player and three offensive players. According to their experimental results, the defensive player required double the time and strokes to score a point than the offensive players did. Moreover, they showed that the playing area of the defensive player was more than double that of the offensive players. Although their study might be outdated for modern table tennis, the results still illustrate that the defensive style is markedly different from the offensive style. The uniqueness of defensive players has often led them to be excluded from research samples (Djokic et al., 2019; Loh and Krasilshchiko, 2015; Malagoli Lanzoni, Di Michele, Baertolomei, \& Semprini, 2019a; Malagoli, katsikadelis, Straub, \& Djokić, 2019b) or analysed separately (Yoshida, Tamaki, \& Yamada, 2019).

Defensive players have been decreasing and offensive styles have become dominant in modern table tennis; however, defensive players remain competitive in world-class table tennis. Defensive players, specifically players who primarily hit a backspin ball by chopping from far from the table, emerged in the late 1930s and dominated until the beginning of 1950 (Straub, 2012). However, defensive players have not won first place in any world championships since 1951. At the beginning of the 1950s, the topspin stroke began to prevail with the evolution of equipment and changes in rules (Straub, 2013). In modern table tennis, the offensive playing style is increasing and currently dominates in both genders, $93.4 \%$ among males and $88.9 \%$ among females (Malagoli Lanzoni et al., 2017). Whereas defensive players have decreased, the defensive style was confirmed to be as competitive as offensive style through an analysis of the number of medals at world championships and world rankings (Straub, 2012). The effectiveness or impact of defensive style should not be underestimated just because it is decreasing.
The purpose of this study was to compare defensive players and offensive players based on the occurrence of missed shots, i.e. shots that scored the opponent, to clarify the influence of playing styles on table tennis matches. While the defensive style is still competitive in modern table tennis, statistical analyses of the defensive style have been limited. Even in a literature review of match analyses in table tennis, no analytical methods or case studies have been described (Fuchs et al., 2018). This study aimed to statistically determine basic differences in the occurrence of missed shots and the number of shots per rally between defensive and offensive styles.

\section{Method}

\section{Match samples}

In the current study, 106 men's singles matches (9029 rallies) and 100 women's singles matches (8268 rallies) were selected from the matches played at the 2012 London Olympic games and the 2016 Rio Olympic games. Table 1 shows the profile of players in the selected matches. Selected matches were categorised as the follows: AA, played by two offensive players, and $\mathrm{AD}$, played by an offensive player and a defensive player. Matches played by two defensive players were excluded from this study because of too small a sample size. Of the selected matches, 94 men's singles matches and 60 women's singles matches were categorised as AA, and 12 men's singles matches and 40 women's singles matches were categorised as AD. In the selected matches, defensive players won in three men's matches and 17 women's matches and lost in 9 men's singles matches and 23 women's matches. The world rankings of the players in the selected matches were from 1 to 131 based on the ranking immediately before each Olympic game. Written informed consent from the subjects was unnecessary as the matches were played in public. 
Table 1.

The profile of players in the selected matches

\begin{tabular}{llccccc}
\hline \multicolumn{1}{c}{ Gender } & \multicolumn{2}{c}{ Playing hand } & \multicolumn{2}{c}{ Playing style } & \multicolumn{2}{c}{ Grip } \\
\hline & $\mathrm{L}$ & $\mathrm{R}$ & Defensive & Offensive & Penhold & Shakehand \\
Male & 63 & 16 & 5 & 74 & 6 & 73 \\
Female & 58 & 20 & 17 & 61 & 5 & 73 \\
\hline
\end{tabular}

\section{Data collection}

The server, winner, and scoring shot number were recorded per rally by observing video recordings broadcast on television or on the Internet. Data were recorded by two operators. If different data were found between the two data collected by the two operators, they reviewed the recordings together, making necessary corrections. Defensive players were identified by their primary use of chop. The agreement rate for the classification was $100 \%$.

\section{Number of shots and missed shots}

The number of shots for each shot number was computed by the method proposed by Tamaki, Yoshida and Yamada (2017). In table tennis, players alternate shots. Thus, we can determine which shot a player hit, if we know the server of the rally and the scoring shot number. Let us assume that player $\mathrm{A}$ serves to player $\mathrm{B}$, and that the seventh shot scores. We can determine that player A performed the first, third, fifth, and seventh shot; player B thus performed the other shots in the rally, including the eighth shot. In this study, the number of shots is defined as the number of shot opportunities. Therefore, the next from the scoring shot is always counted as the missed shot, regardless of whether it was performed. In the aforementioned example, the eighth shot is the missed shot. The number of missed shots was counted for each shot number.

\section{Occurrence of missed shots and number of shots per rally}

The occurrence of missed shots was calculated as a measure of defensiveness. Moreover, the number of shots per rally was calculated as a measure of rally length. In fact, the occurrence of missed shots and the number of shots per rally are reciprocals of each other and provide identical information. However, the number of shots per rally was calculated to easily see the rally length. The occurrence of missed shots and the number of shots per rally were calculated for each player in each match.

\section{Occurrence of missed shots at each shot number}

The occurrence of missed shots was calculated for each shot number. Let $m_{i}$ be the number of missed shots at the $i$-th shot and $s_{i}$ the number of $i$-th shots. The occurrence of missed shots at the $i$-th shot was calculated as $m_{i} / s_{i}$. The fifth and the subsequent shots were combined as one group, and the occurrence of missed shots was calculated by the following equation.

$$
\% \text { Miss }_{5+}=\sum_{i=5}^{n} m_{i} / \sum_{i=5}^{n} s_{i}
$$

The occurrence of missed shots of each shot number was calculated for each player in each match.

\section{Precision and recall of automatic classification}

Precision and recall of automatic clustering were calculated to measure how defensive players differed from offensive players. A Gaussian mixture model was used to statistically model the distribution. Twogroup clustering was performed with the occurrence of missed shots at third shot and at the fifth shot using the expectation-maximisation algorithm (Bishop, 2006). Let TP be the number of true positives, FP the number of false positives, and FN the number of false negatives. Precision and recall of automatic classification were calculated using the following equations. 


$$
\text { Precision }=T P /(T P+F P)
$$

$$
\text { Recall }=T P /(T P+F N)
$$

\section{Statistical analysis}

The occurrence of missed shots was compared between AA and AD using the Mann-Whitney U test. In addition, a Kruskal-Wallis test was performed to compare the occurrence of missed shots among the following combinations of server and receiver playing styles: A-A, an offensive player served and an offensive player returned it; A-D, an offensive player served and a defensive player returned it; and D-A, a defensive player served and an offensive player returned it. Wherever significant differences were observed, a Dunn test with Bonferroni adjustment was used to compare the categories of the rally. All statistical tests were performed for gender at a $95 \%$ confidence level. In addition, every statistic was also compared with effect size, Pearson r, and 95\% confidential interval. We analysed the magnitude of $r$ with reference to the general frame proposed by Cohen (1988) as follows:

Small: $r=0.1$

Medium: $r=0.3$

Large: $r=0.5$.

\section{Results}

Occurrence of missed shots and number of shots per rally

Figure 1 shows the occurrence of missed shots and the number of shots per rally by match category. The occurrence of missed shots in $\mathrm{AD}$ was significantly lower than in AA (male: $\mathrm{p}<0.01, \mathrm{r}=0.36$; female: $\mathrm{p}<0.01, \mathrm{r}=0.72$ ). Hence, as a matter of course, AD's number of shots per rally, namely the reciprocal of the occurrence of missed shots, was larger than that of AA.

\section{Occurrence of missed shots at each shot number}

Figure 2 shows the occurrence of missed shots at each shot number. The occurrence of missed shots for $\mathrm{AD}$ was lower than for $\mathrm{AA}$ at the $2 \mathrm{nd}$ shot (male: $\mathrm{p}<0.01, \mathrm{r}=0.22$; female: $\mathrm{p}<0.01, \mathrm{r}=0.51$ ), $3 \mathrm{rd}$ shot (male: $\mathrm{p}<0.01, \mathrm{r}=0.37$; female: $\mathrm{p}<0.01, \mathrm{r}=0.58$ ), 4 th shot (male: $\mathrm{p}<0.01, \mathrm{r}=0.27$; female: $\mathrm{p}<0.01, \mathrm{r}=0.71$ ), and after the 4th shot (male: $\mathrm{p}<0.01, \mathrm{r}=0.37$; female: $\mathrm{p}<0.01, \mathrm{r}=0.71)$. The occurrence of missed shots at the 1st shot for $\mathrm{AD}$ was not significantly different from that of AA (male: $\mathrm{p}=0.15, \mathrm{r}=0.10$; female: $\mathrm{p}=0.26, \mathrm{r}=0.08$ ). Figure 3 shows the cumulative occurrence of shots per shot number. AD's cumulative occurrence of shots surpassed 0.75 at the 8th shot in male matches and at the 11th shot in female matches, while that of AA surpassed 0.75 at the 5 th shot in both male and female matches.

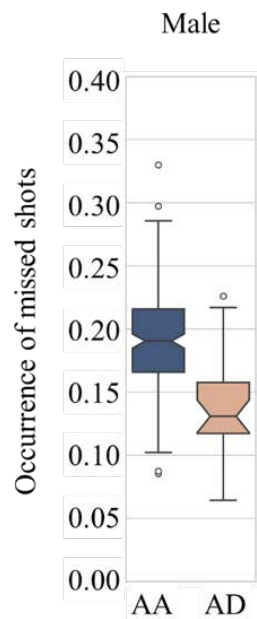

(a)

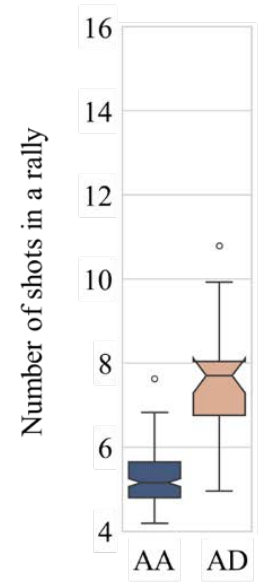

(c)

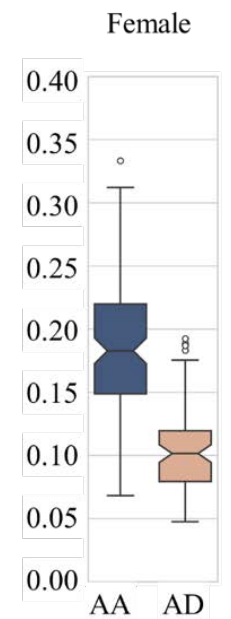

(b)

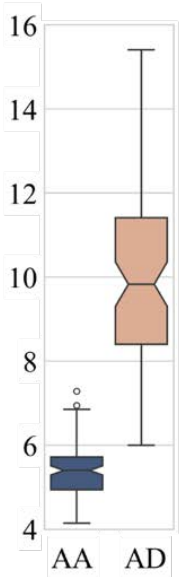

(d)
Figure 1. Occurrence of missed shots and number of shots per rally by match category, "AA," played by two offensive players, and "AD," played by an offensive player and a defensive player. The box and 
whiskers denote the maximum, minimum, median, first quartile and third quartile. The notches denote the $95 \%$ confidence interval. The circle markers denote outliers whose distance from the box is at least 1.5 times the inter quartile range.

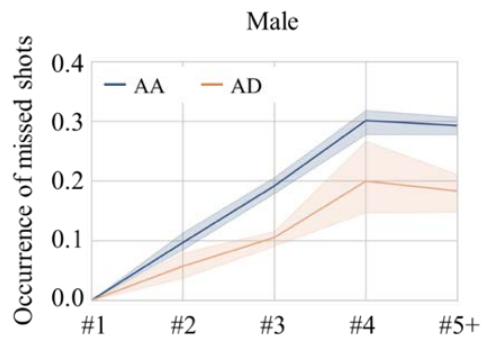

(a)

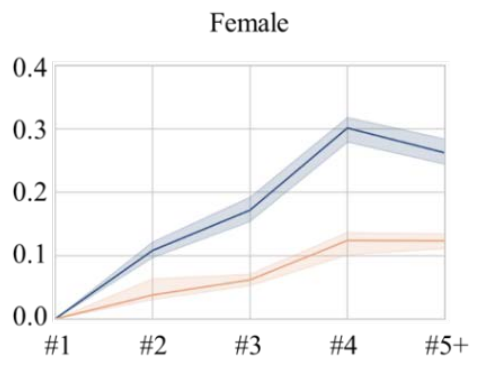

(b)

Figure 2. Occurrence of missed shots at each shot number. "\#i" denotes the $i$-th shot. The median was calculated for each gender. The shaded area denotes the $95 \%$ confidence interval.

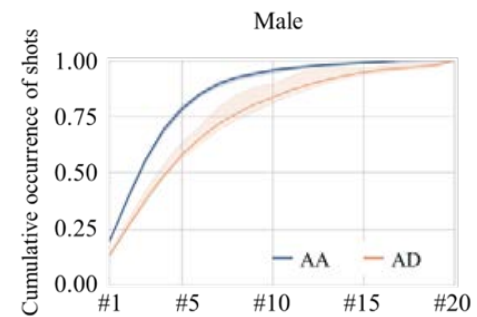

(a)

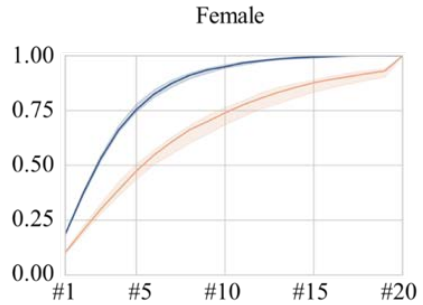

(b)
Figure 3. Cumulative occurrence of shots per shot number. "\#i" denotes the $i$-th shot. The median was calculated for each gender. The shaded area denotes the $95 \%$ confidence interval.

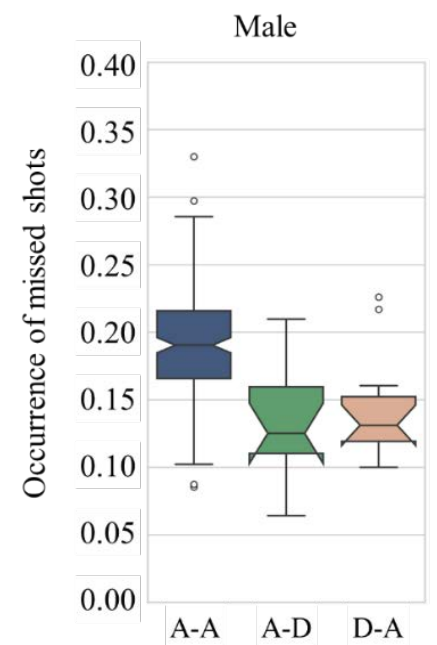

(a)

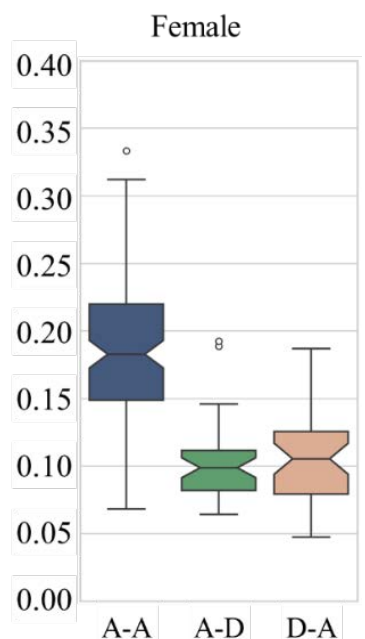

(b)
Figure 4. Occurrence of missed shots for each combination of the server's and receiver's playing style: "A-A," an offensive player served and an offensive player returned it; "A-D," an offensive player served and a defensive player returned it; and "D-A," a defensive player served and an offensive player returned it. The box and whiskers denote the maximum, minimum, median, first quartile and third quartile. The notches denote the $95 \%$ confidence intervals. The circle markers denote outliers, whose distance from the box is at least 1.5 times the inter quartile range.

\section{Occurrence of missed shots for each combination of server and receiver playing styles}

Figure 4 shows the occurrence of missed shots of each combination of server and receiver playing styles. The occurrence of missed shots for A-A was significantly higher than that for A-D (male: $p=$ $0.00, r=0.29$; female: $p=0.00, r=0.66$ ) and D-A (male: $p=0.00, r=0.25$; female: $p=0.00, r=0.61$ ). A-D's occurrence of missed shots was not significantly different from that of D-A (male: $p=$ 1.00, $r=0.08$; female: $p=1.00, r=0.06$ ). 


\section{Male}

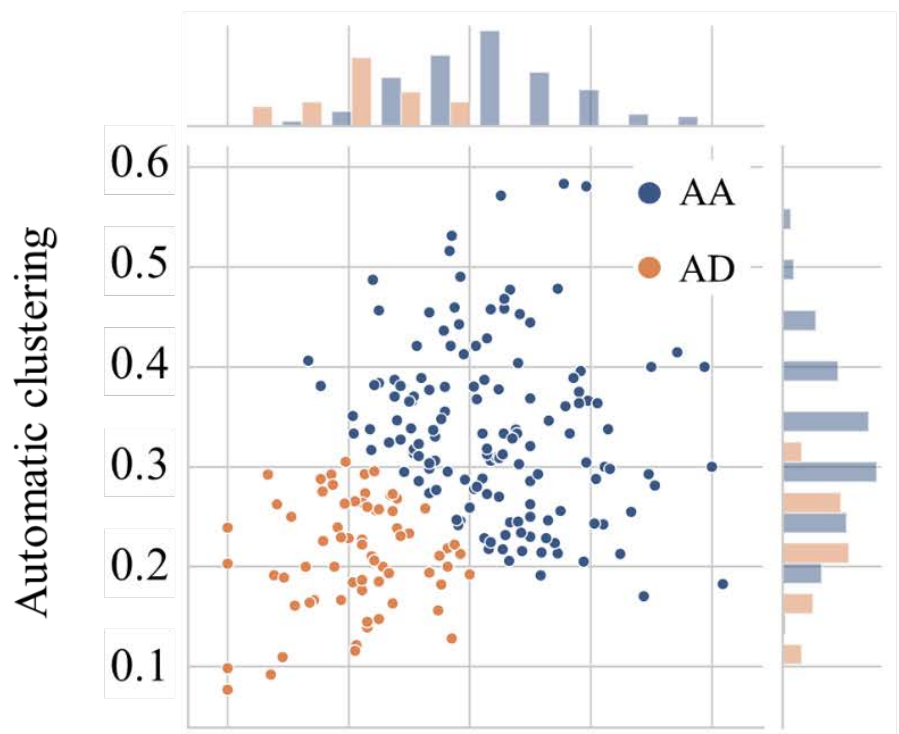

(a)

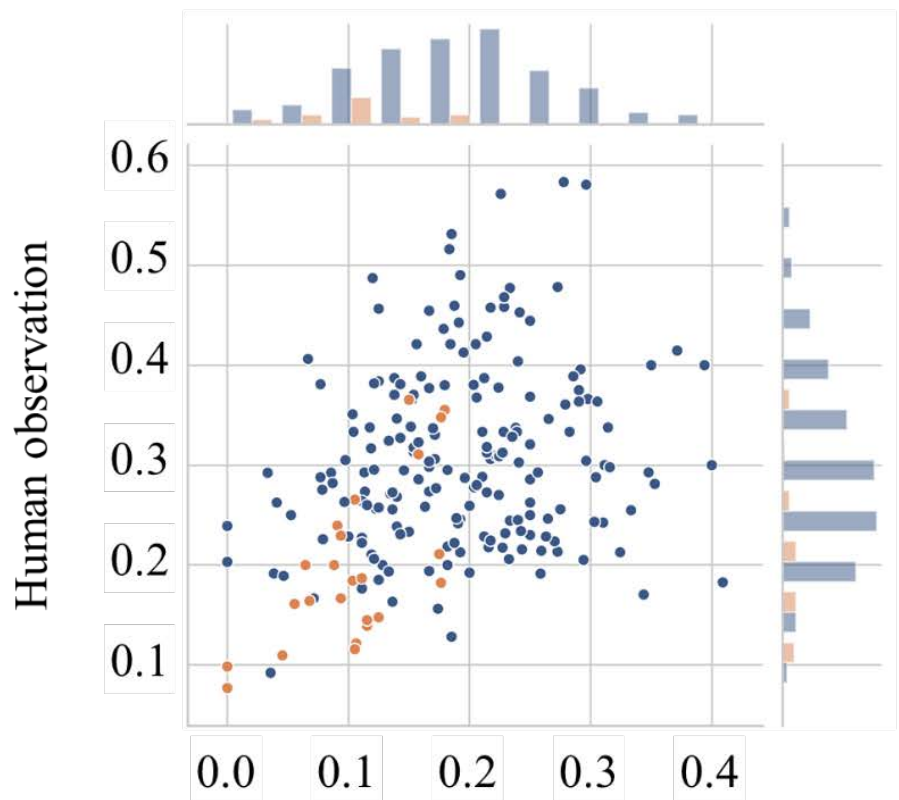

(c)
Female

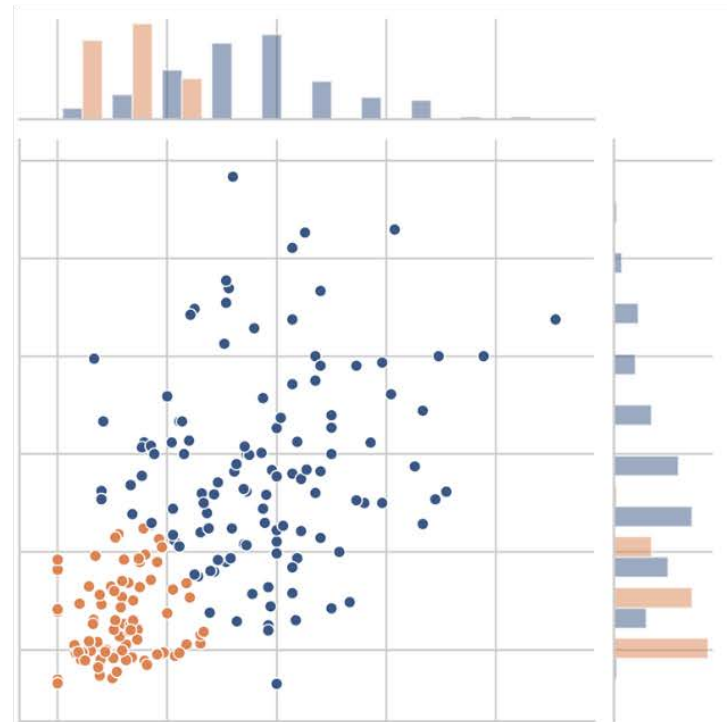

(b)

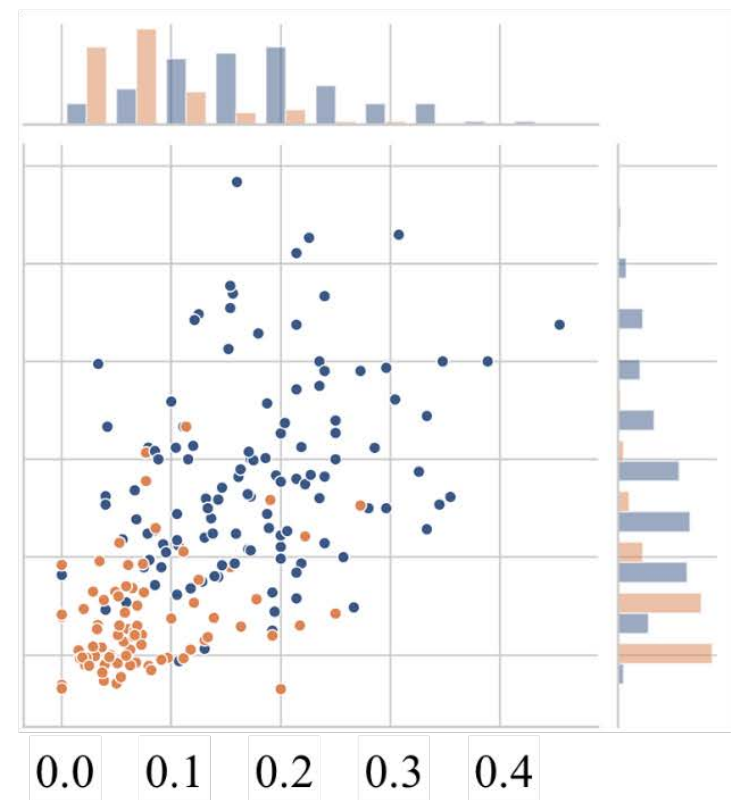

(d)

Figure 5. The results of clustering based on the occurrence of missed shots at the third shot (horizontal axis) and the fifth shot (vertical axis). Automatic clustering denotes the results of Gaussian Mixture Model (GMM)-based clustering, and Human observation denotes the data labelled through human observation. 


\section{Precision and recall of automatic classification}

Table 2 shows the precision and recall of the automatic classification. Figure 5 shows the distribution of the occurrence of missed shots at the 3 rd shot and the 5th shot with the category of match automatically labelled and labelled through human observation. The precision and recall were 0.29 (20 / $70)$ and 0.83 (20/24) in male players' matches and $0.80(63 / 79)$ and $0.79(63 / 80)$ in female players' matches.

Table 2.

Precision and recall of automatic clustering. TP denotes the number of true positives, TF denotes the number of true negatives, FP denotes the number of false positives, and FN denotes the number of false negatives.

\begin{tabular}{lrrlllr}
\hline Gender & Precision & Recall & TP & TF & FP & FN \\
\hline Male & 0.29 & 0.83 & 20 & 138 & 50 & 4 \\
Female & 0.80 & 0.79 & 63 & 104 & 16 & 17 \\
\hline
\end{tabular}

\section{Discussion}

\section{Influence of playing style on the occurrence of missed shots}

The defensive style was less likely to lead to missed shots in both male and female players' matches. A rough calculation shows that the difference in the occurrence of missed shots between $\mathrm{AA}$ and $\mathrm{AD}$ was about $6 \%$ in male matches, and $8 \%$ in female matches. The difference in the occurrence of missed shots increased by 2.5 shots in male matches, and by 4.4 shots in female matches. This indicates the influence of playing style on the occurrence of missed shots. These results can be naturally described by defensive players' tactics, namely winning a point by lowering one's losing rate more than one's opponent's. Although these results were not surprising, specific differences between the two categories of match were novel in research on table tennis because researchers commonly exclude defensive players in their research sample (Djokic et al., 2019; Loh and Krasilshchiko, 2015; Malagoli
Lanzoni et al., 2019a; Malagoli et al., 2019b). These results are therefore meaningful for understanding the influence of playing style on table tennis rally in a quantitative way.

Even if a rally was initiated by an offensive player's service, the occurrence of missed shots would be as low as in the rallies begun by a defensive player's service. The effect size between A-D and D-A was 0.08 in male matches, and 0.06 in female matches, which can be interpreted as small or less than small according to Cohen's guidelines. If offensive players take advantage of service in their tactics, which is more likely than with defensive players, high missed shots ratio at A-D and low missed shots ratio at D-A could co-occur because A-D and D-A are independent. Although the specific reasons cannot be clarified in the current study, these results indicate that the defensive style also reduces missed shots in a rally served by an offensive player, and the amount of reduction is about the same as in a rally served by a defensive player.

\section{Changes in the influence of playing style on the occurrence of missed shots}

The defensive style lowers the occurrence of missed shots, especially after the fourth shot, but also at the second and following shots. According to previous studies (Tamaki et al., 2017; Yoshida et al., 2014; Zhang et al., 2014), servers are considered to have the potential advantage of scoring bias toward themselves using service in table tennis. Therefore, if defensive players use services in the same manner as offensive players, it can be naturally inferred that similar amounts of missed shots occurred immediately after the service, such as at the second and third shots. However, the differences in effect size between $\mathrm{AD}$ and $\mathrm{AA}$ at the second and third shots revealed that missed shots were less likely to occur in $\mathrm{AD}$ than in $\mathrm{AA}$, even at the second and third shots. On the other hand, the degree of influence of defensive style was notably different between the second shot and shots after the fourth shot. Missed shots were more likely to occur after the fourth shot than the second shot. Consequently, we can understand that defensive style decreases the 
incidence of missed shots particularly after the fourth shot, although the incidences at the second and the following shots are also decreased.

\section{Gender comparison}

The occurrence of missed shots at each shot number suggest that the tactics of male defensive players is less defensive than those of female defensive players. According to the effect size, the difference between AA and AD was medium in male matches and large in female matches. Since the $95 \%$ confidence intervals of AA in female and male matches overlapped (Figure 1), we can reject the hypothesis that missed shots are less likely to occur in matches played by female offensive players. Hence, we can hypothesise that missed shots are more likely to occur in male players' $\mathrm{AD}$ than female players' AD. The occurrence of missed shots at each shot number provides detailed insight of this hypothesis. In male players' matches, the occurrences of missed shots in $\mathrm{AD}$ and AA were relatively similar at the fourth shot according to the effect size, 0.37 at the third shot, 0.27 at the fourth shot, and 0.36 at the shots after the fourth shot. This suggests that male defensive players also use service to score a point at the third shot and the ratio of missed shots at the fourth shot was close to that of the matches played by offensive players. On the other hand, such results were not observed in female players' matches; the effect size was 0.58 at the third shot, 0.71 at the fourth shot, and 0.71 at the fifth shot. This suggests that female offensive players are less likely to make a missed shot at the third shot in AD. The specific factors of the difference cannot be mentioned in this study because information about the technique (e.g. kind of shot, ball placement) were not collected. However, the results suggest a gender difference in which the tactics of male defensive players are less defensive than those of female defensive players.

\section{Can we distinguish between defensive and offensive} players?

It was found that we could not precisely identify defensive and offensive players by the occurrence of missed shots. According to precision and recall of automatic clustering, if we try to detect defensive players by automatic clustering, we will have a $20 \%$ chance of accidentally finding matches played by offensive players in the female cases and a $70 \%$ chance of finding matches played by offensive players in the male case. The difficulty of clustering can be understood from Figure 5; there is no division in the distribution of the occurrence of missed shots. The results suggested the necessity of reconsidering the definition of "defensive player" in table tennis. In this study and other studies (Djokic et al., 2019; Malagoli Lanzoni et al., 2019a; Malagoli Lanzoni et al., 2019b; Straub, 2012), the definition of a defensive player is the same as that of choppers. According to the results of this study, we may say that defensiveness or returning stability are not just determined by how often players perform a chop. In the distribution of missed shots, there seem to be a remarkable number of players whose defensiveness was equal to or higher than that of choppers. However, such players cannot be defensive under the current definition because the definition requires the frequent use of chopping. While acknowledging that choppers are relatively defensive, we need to reconsider the definition of "defensive player" based on the fact that there are other types of defensive players than choppers.

\section{Suggestions for athletes and coaches}

This study presents useful information for planning technical or tactical practice and physical training. From the cumulative occurrence of shots per shot number, more than $50 \%$ of rallies last more than five shots in female players' matches. Female defensive players and their coaches can understand the importance of the tactics and techniques to score a point at shots after the fifth shot from this result. In addition, as the number of shots per rally is almost twice as many, athletes and coaches can understand 
the reasonable amount of physical training required. Furthermore, defensiveness is not determined by frequency of chopping. The occurrence of missed shots, or number of shots per rally, should be utilized to understand defensiveness of opponent players to formulate effective tactics to score a point. Thus, athletes and coaches can gain useful information from this study.

\section{Limitation of this study}

Our findings have some limitations. As this study focused on the occurrence of missed shots in table tennis, the information about techniques, such as kind of shot or ball placement, were not collected. Therefore, no specific techniques in the analysis were mentioned. For example, the difference in the occurrence of missed shots between A-D and D-A was found to be small in this study. Possible reasons for this result: offensive players might change their tactics when they play with defensive players, defensive players might sufficiently reduce server advantage with their defensive techniques, and so on. However, specific reasons were not determined as no information about playing techniques was included.

\section{Conclusion}

The purpose of this study was to clarify the influence of playing style on the occurrence of missed shots in table tennis. We found that matches played by a defensive player had a $6-8 \%$ lower chance of making missed shots. Further investigation revealed that defensive style decreases the occurrence of missed shots particularly after the fourth shot, although also at the second and following shots. In addition, the defensive style also reduces missed shots in a rally served by an offensive player, and the amount of reduction is about the same as for a rally served by a defensive player. The occurrence of missed shots was compared between genders, showing that male defensive players were less defensive than female defensive players. It was also found that we cannot precisely identify defensive players based only on the occurrence of missed shots because there may be players who were not categorised as defensive players but who were as defensive as defensive players.

\section{References}

Bishop, M. (2006). Pattern recognition and machine learning (pp. 430-439). New York: Springer.

Cohen, J. (1988). Statistical power analysis for the behavioral sciences second edition (pp.215-271). New Jersey: Lawrence Erlbaum Associates.

Djokic, Z., Straub, G., Malagoli Lanzoni, I., Katsikadelis, M., \& Munivrana, G. (2019). Effects of rule changes on performance efficacy: differences between winners and losers table tennis players. Facta Universitatis Series Physical Education and Sport, 17(1), 149-163.

Fuchs, M., Liu, R., Malagoli Lanzoni, I., Munivrana, G., Straub, G., Tamaki, S., Yoshida, K., Zhang, H., \& Lames, M. (2018). Table tennis match analysis: a review. Journal of Sports Sciences, 36, 26532662.

Loh, T.C., \& Krasilshchikov, O. (2015). Competition performance variables differences in elite and U-21 international men singles table tennis players. Journal of Physical Education and Sport, 15(4), 829-833.

Malagoli Lanzoni, I., Di Michele, R., Bartolomei, S., \& Semprini, G. (2019a). Do left-handed players have a strategic advantage in table tennis? International Journal of Racket Sports Science, 1(1), 6169.

Malagoli Lanzoni, I., Di Michele, R., \& Semprini, G. (2017). Fifty years of top-class table tennis world ranking. In Kondrič, M., Fuchs, M., Matjašič, T. (Eds.). Proceedings book of the $15^{\text {th }}$ ITTF Sports Science

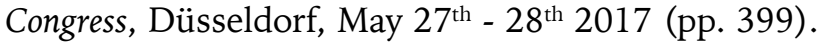
Lausanne: International Table Tennis Federation.

Malagoli Lanzoni, I., Katsikadelis, M., Straub, G., \& Djokić, Z. (2019b). Footwork technique used in elite table tennis matches. International Journal of Racket Sports Science, 1 (2), 44-48.

Straub, G. (2012). In the beginning was the halfvolley: the history of defence play in table tennis part I. The Table Tennis Collector, 63, 16-21.

Straub, G. (2013). The spreading and playing ability of defensive players in competitive table 
tennis. International Journal of Table Tennis Sciences, 8, 179-183.

Tamaki, S., Yoshida, K., \& Yamada, K. (2017). A shot number based approach to performance analysis in table tennis. Journal of Human Kinetics, 55, 7-18.

Yoshida, K., Tamaki, S., \& Yamada, K. (2019). Comparison of top-level world table tennis rallies in Rio and London Olympic games. In Kondrič, M., Paar, David (Eds.). Book of abstracts of the $16^{\text {th }}$ ITTF Sport Science Congress, Budapest, 19th $-20^{\text {th }}$ April 2019, (pp. 73). Pécs: University of Pécs: International Table Tenis Federation: Hungarian Table Tenis Association.

Yoshida, K., Yamada, K., Tamaki, S., Naito, H., \& Kaga, M. (2014). Characteristics of world-class rally in table tennis: focusing on the number of shots played per rally. The Japan Journal of Coaching Studies, 28(1), 65-74.

Yuza, N., Sasaoka, K., Nishioka, N., Matsui, Y., Yamanaka, N., Ogimura, I., Takashima, N., \& Miyashita, M. (1992). Game analysis of table tennis in top Japanese players of different playing styles. International journal of table tennis sciences, 1, 7989.Zhang, H., Liu, W., Hu, J.-J., \& Liu, R.Z. (2014). Evaluation of elite table tennis players' technique effectiveness. Journal of Sports Sciences, 32(1), 70-77. 